\title{
SCALAR CURVATURE AND WARPED PRODUCTS OF RIEMANN MANIFOLDS
}

\author{
F. DOBARRO AND E. LAMI DOZO
}

\begin{abstract}
We establish the relationship between the scalar curvature of a warped product $M \times_{f} N$ of Riemann manifolds and those ones of $M$ and $N$. Then we search for weights $f$ to obtain constant scalar curvature on $M \times{ }_{f} N$ when $M$ is compact.
\end{abstract}

1. Introduction. Let $M=\left(M_{m}, g\right)$ and $N=\left(N_{n}, h\right)$ be two Riemann manifolds. For $f \in C^{\infty}(M), f>0$ on $M$, we consider the warped product $M \times_{f} N=\left((M \times N)_{m+n}, g+f^{2} h\right)$ and show the relationship between the scalar curvatures $R$ on $M, H$ on $N$ and $\tilde{R}$ on $M \times_{f} N$. This relationship is a nonlinear partial differential equation satisfied by a power of the weight $f$. In the case $M$ is compact and connected, we obtain a geometric interpretation of the principal eigenfunction and eigenvalue of the canonical elliptic operator $-\Delta+R / 2$, where $\Delta$ denotes the laplacian on $M$. Finally we consider the question of finding a weight $f$ such that $M \times_{f} N$ has constant scalar curvature. This question is equivalent to find a positive solution to a nonlinear eigenvalue problem.

The notion of warped product $M \times_{f} N$ generalizes that of a surface of revolution. It was introduced in [B-O] for studying manifolds of negative curvature (cf. $[\mathbf{Z}]$ for other applications). The Riemann metric $\tilde{g}=g+f^{2} h$ on $M \times_{f} N$ is defined for pairs of vector fields $\tilde{X}, \tilde{Y}$ on $M \times N$ by

$$
\tilde{g}(\tilde{X}, \tilde{Y})=g\left(\pi_{*} \tilde{X}, \pi_{*} \tilde{Y}\right)+f^{2}(\pi(\cdot)) h\left(\omega_{*} \tilde{X}, \omega_{*} \tilde{Y}\right)
$$

where $\pi$ and $\omega$ are the canonical projections over $M$ and $N$ respectively.

We denote by $\Delta_{g}$ or $\Delta$ the laplacian (or Laplace-Beltrami) operator on $\left(M_{m}, g\right)$ with local expression $\Delta_{g} u=\nabla^{i} \nabla_{i} u=|g|^{-1 / 2} \partial_{i}\left(g^{i j}|g|^{1 / 2} \partial_{j} u\right)$, for $u \in C^{2}(M)$ (cf. [Au1, B-G-M]). Thus $\Delta u=u^{\prime \prime}$ for a real valued function $u$ on $M=\mathbf{R}$.

2. The equation. Given a metric $g^{\prime}=k g$ with $k \in C^{\infty}(M), k>0$ on $M, g^{\prime}$ is said to be conformal to $g$. It is known that the scalar curvature $R^{\prime}$ on $\left(M_{m}, g^{\prime}\right)$ is related to $R$, the one on $\left(M_{m}, g\right)$, by the Yamabe equation

$$
-\frac{4(m-1)}{m-2} \Delta_{g} u+R u=R^{\prime} u^{(m+2) /(m-2)}
$$

where $k=u^{4 /(m-2)}$, whenever $m \geq 3[\mathbf{4}, \mathbf{A u 1}$, p. 126].

Received by the editors July 15, 1986.

1980 Mathematics Subject Classification. Primary 58G30, 53C25, 58C40; Secondary 47H12, $47 \mathrm{H} 15$.

Key words and phrases. Nonlinear eigenvalue problem, principal eigenvalue and eigenfunction, bifurcation from infinity. 
If we consider now the case of a warped product we have

THEOREM 2.1. Let $R, H$ and $\tilde{R}$ denote the scalar curvature on $M, N$ and $M \times_{f}$ $N$ respectively. Then the following equality holds:

$$
-\frac{4 n}{n+1} \Delta_{g} u+R u+H u^{(n-3) /(n+1)}=\tilde{R} u
$$

where $u=f^{(n+1) / 2}$.

PROOF. We write $\tilde{g} \equiv g+f^{2} h=f^{2}\left(f^{-2} g+h\right)$, so $\tilde{g}$ is conformal to $\widetilde{g}=f^{-2} g+h$ on $M \times N$ and $f^{-2} g$ is conformal to $g$ on $M$.

Supposing $m \geq 3$, we apply (Ya) in $M$ to obtain that $f$ satisfies

$$
-\frac{4(m-1)}{m-2} \Delta_{g} \eta+R \eta=\eta^{(m+2) /(m-2)} \hat{R}
$$

with $\eta^{4 /(m-2)}=f^{-2}$ and where $\hat{R}$ denotes the scalar curvature on $\left(M_{m}, f^{-2} g\right)$. As $m+n \geq 3$, we use (Ya) in $M \times N$. Hence $f$ also satisfies

$$
-\frac{4(m+n-1)}{m+n-2} \Delta_{\widetilde{g}} \psi+\widetilde{\widetilde{R}}_{\psi}=\tilde{R} \psi^{(m+n+2) /(m+n-2)}
$$

with $\psi^{4 /(m+n-2)}=f^{2}$, where $\widetilde{\widetilde{R}}$ denotes the scalar curvature on $\left((M \times N)_{m+n}, \widetilde{\widetilde{g}}\right)$ and $\Delta_{\widetilde{g}}$ the corresponding laplacian.

From $\psi \in C^{\infty}(M)$ we deduce that $\Delta_{\widetilde{g}} \psi \equiv \Delta_{f^{-2} g+h} \psi=\Delta_{f^{-2} g} \psi$. Working in local coordinates

$$
\Delta_{f^{-2} g} \psi=\left|f^{-2} g\right|^{-1 / 2} \partial_{i}\left[\left(f^{-2} g\right)^{i j}\left|f^{-2} g\right|^{1 / 2} \partial_{j} \psi\right]
$$

with $\left|f^{-2} g\right|=\operatorname{det}\left(f^{-2} g_{i j}\right)=f^{-2 m}|g|$ and $\left(f^{-2} g\right)^{i j}=f^{2} g^{i j}$. Hence

$$
\begin{aligned}
\Delta_{f^{-2} g} \psi & =\eta^{-2 m /(m-2)}|g|^{-1 / 2} \partial_{i}\left[\eta^{2} g^{i j}|g|^{1 / 2} \partial_{j} \psi\right] \\
& =\left[\eta \Delta_{g} \psi+2 g^{i j} \partial_{i} \eta \partial_{j} \psi\right] \eta^{-(m+2) /(m-2)} .
\end{aligned}
$$

On the other hand

$$
\Delta_{g}(\eta \psi)=\eta \Delta_{g} \psi+\psi \Delta_{g} \eta+2 g^{i j} \partial_{i} \eta \partial_{j} \psi
$$

and from (2.4) we get

$$
\eta^{(m+2) /(m-2)} \Delta_{\widetilde{g}} \psi=\Delta_{g}(\eta \psi)-\psi \Delta_{g} \eta
$$

We have that $\widetilde{R}=\hat{R}+H$, because we consider the usual product of $\left(M_{m}, f^{-2} g\right)$ by $\left(N_{n}, h\right)$. Using this in (2.3), multiplying by $\eta^{(m+2) /(m-2)}$, we obtain from $(2.5)$

$$
\begin{gathered}
-\frac{4(m+n-1)}{m+n-2}\left(\Delta_{g}(\eta \psi)-\psi \Delta_{g} \eta\right)+\eta^{(m+2) /(m-2)}(\hat{R}+H) \psi \\
=\tilde{R} \psi^{(m+n+2) /(m+n-2)} \eta^{(m+2) /(m-2)} .
\end{gathered}
$$

From (2.2) we arrive at

$$
\begin{gathered}
-\frac{4 n}{(m+n-2)(m-2)} \psi \Delta_{g} \eta-\frac{4(m+n-1)}{(m+n-2)} \Delta_{g}(\eta \psi)+R \eta \psi+H \eta^{(m+2) /(m-2)} \psi \\
=\tilde{R} \psi \eta \psi^{4 /(m+n-2)} \eta^{4 /(m-2)} .
\end{gathered}
$$


Recalling that $\psi^{4 /(m+n-2)} \eta^{4 /(m-2)}=1$, denoting $u=f^{(n+1) / 2}$, replacing in terms of $u$ in this last equality, then multiplying by $u^{1-n /(n+1)}$, we obtain

$$
\left\{\begin{array}{l}
-\frac{4 n}{(m+n-2)(m-2)} u^{(m+n-1) /(n+1)} \Delta_{g} u^{-(m-2) /(n+1)} \\
-\frac{4(m+n-1)}{m+n-2} u^{1 /(n+1)} \Delta_{g} u^{n /(n+1)}+R u+H u^{(n-3) /(n+1)}=\tilde{R} u .
\end{array}\right.
$$

For any $\alpha \neq 0, v \in C^{\infty}(M), v>0$ on $M$ satisfies

$$
\Delta_{g} v^{\alpha}=\alpha(\alpha-1) v^{\alpha-2} \nabla^{i} v \nabla_{i} v+\alpha v^{\alpha-1} \Delta_{g} v .
$$

Choosing first $\alpha=n /(n+1)$, then $\alpha=-(m-2) /(n+1)$ in $(2.7)$ (for $v=u)$ we obtain from (2.6) the desired result (2.1).

REMARK. Formula (2.1) holds even for $m=1,2$. For $m=1$, it is easily deduced from a similar formula in $[\mathbf{E - 1}]$. For $m=2$, the proof is similar but Yamabe equation is for the conformal change $g^{\prime}=e^{u} g$ :

$$
-\Delta_{g} u+R=R^{\prime} e^{u}
$$

(cf. [M, Au1, p. 119]). (2.1) could also be deduced from a formula in [B-O, p. 26] but this formula is a consequence of an unwritten 15-term calculation and besides our method of proof is different.

3. Constant scalar curvature. Let the scalar curvatures $R$ of $M$ and $H$ of $N$ be fixed, we look for a weight $f \in C^{\infty}(M), f>0$, on $M$ such that the warped product $M \times_{f} N$ has constant scalar curvature $\tilde{R}$ and then which constants are attained. Taking account of $R \in C^{\infty}(M), u=f^{(n+1) / 2} \in C^{\infty}(M)$ and $H \in$ $C^{\infty}(N)$, it follows easily from Theorem 2.1 that for $\tilde{R}$ to be a constant $\lambda$, it is necessary that $N$ have constant scalar curvature, still denoted $H$.

The simplest case is $H=0$.

THEOREM 3.1. Let $M$ be compact and connected. Suppose $N$ of zero scalar curvature. Then there exists a weight $f$ such that the scalar curvature $\tilde{R}$ on $M \times_{f} N$ is a constant $\lambda_{1} . f$ is unique up to a positive multiplicative constant, $\lambda_{1}$ is unique and is given by

$$
\lambda_{1}=\inf \left\{\int_{M}\left(\frac{4 n}{n+1}|\nabla v|^{2}+R v^{2}\right) d V ; v \in H^{1}(M), \int_{M} v^{2} d V=1\right\}
$$

where $H^{1}(M)=\left\{v \in L^{2}(M) ;|\nabla v|^{2} \equiv \nabla^{i} v \nabla_{i} v \in L^{1}(M)\right\}$ is the Sobolev space of order 1 .

PROOF. From (2.1) we deduce that we search $\lambda \in \mathbf{R}$ and $u \in C^{\infty}(M), u>0$ on $M$ such that

$$
L u=\lambda u \text { on } M
$$

where $L u=-4 n \Delta_{g} u /(n+1)+R u$.

It is well known that this linear eigenvalue problem on $M$ compact and connected has only one nonnegative solution $u_{1}$ with $\max _{M} u_{1}=1$; cf. [Au1, p. 137] (in fact $u_{1}>0$ on $\left.M\right) u_{1}$ is the so-called principal eigenfunction of the elliptic operator $L$. The corresponding eigenvalue $\lambda_{1}$ is simple and is called the principal eigenvalue. 
Hence $f=u_{1}^{2 /(n+1)}>0$ on $M$ is the weight we are searching. Any other solution is of the form $r f, r \in \mathbf{R}_{0}^{+}$, because $\lambda_{1}$ is simple. The formula for $\lambda_{1}$ is classical.

The case of warping $M$ with a circle, i.e. $M \times_{f} S^{1}$ gives a geometric interpretation of the principal eigenfunction $u_{1}$ of $-\Delta+R / 2$ and its corresponding eigenvalue $\lambda_{1}$, which in the special case of $M$ 2-dimensional can be expressed in terms of the classical Gaussian curvature on $M$.

COROLlARY. Given a compact, connected $M=\left(M_{2}, g\right)$ with Gaussian curvature $K$ and Laplace-Beltrami operator $\Delta$, then the principal eigenfunction $u_{1}$ of the canonical elliptic operator $-\Delta+K$ holds the property that the warped product $M \times_{u_{1}} S^{1}$ has a scalar curvature constantly equal to the principal eigenvalue $\lambda_{1}$ of $-\Delta+K$.

ProOF. Take $n=1$ in (2.1). Recall that $H=0$ in $S^{1}$ and $R / 2=K$ in a 2-dimensional manifold.

The case $H \in \mathbf{R}, H<0$ is similar to $H=0$ because the half-line $\left\{\left(\lambda_{1}, r u_{1}\right)\right.$, $r>0\}$ is deformed into a curve $\left\{(\lambda, f(\lambda)) ; \lambda<\lambda_{1}\right\}$ :

THEOREM 3.2. Let $M=\left(M_{m}, g\right)$ be compact and connected. Suppose $N=$ $\left(N_{n}, h\right)$ of constant negative scalar curvature $H$ and assume $n \geq 3$. Let $\lambda_{1}$ denote the principal eigenvalue of $-4 n \Delta_{g} /(n+1)+R$. Then for each $\lambda<\lambda_{1}$ there exists a unique weight $f=f(\lambda)$ such that $M \times_{f} N$ has constant scalar curvature $\lambda . N o$ constant $\geq \lambda_{1}$ may be curvature of $M \times{ }_{f} N$ for any weight $f$.

PROOF. We look for positive solutions $u$ in $C^{\infty}(M)$ of

$$
L u+H u^{\alpha}=\lambda u \text { on } M
$$

where $L u=-4 n \Delta_{g} u /(n+1)+R u, 0 \leq \alpha=(n-3) /(n+1)<1($ cf. $(2.1))$.

Let us still denote $u_{1}$ the positive eigenfunction of $L u=\lambda u$ but with $L^{2}$-norm 1, i.e. $\int_{M} u_{1}^{2} d V=1$. If $u$ is a positive solution of (3.2), then multiplying (3.2) by $u_{1}$ and integrating by parts ( $L$ is selfadjoint), we obtain

$$
\lambda_{1} \int_{M} u u_{1} d V+H \int_{M} u^{\alpha} u_{1} d V=\lambda \int_{M} u u_{1} d V
$$

Hence

$$
\left(\lambda-\lambda_{1}\right) \int_{M} u u_{1} d V=H \int_{M} u^{\alpha} u_{1} d V
$$

Then $H<0$ necessarily gives $\lambda<\lambda_{1}$.

Let us fix $\lambda<\lambda_{1}$. As $0 \leq \alpha<1$, we have that

$$
(L-\lambda I) \underline{t} u_{1} \leq|H| \underline{t}^{\alpha} u_{1}^{\alpha}
$$

for $\underline{t} \in \mathbf{R}^{+}$small enough, so $\underline{t} u_{1}$ is a subsolution of (3.2). Also

$$
(L-\lambda I) \bar{t} u_{1} \geq|H| \bar{t}^{\alpha} u_{1}^{\alpha}
$$

for $\bar{r} \in \mathbf{R}^{+}$big enough, so we have a supersolution $\bar{t} u_{1} \geq \underline{t} u_{1}$.

The operator $(L-\lambda I): C^{2, \beta}(M) \rightarrow C^{\beta}(M)$ is an isomorphism and its inverse $(L-\lambda I)^{-1}$ is continuous for the $C^{0}$-norm on $C^{\beta}(M)$ and the $C^{1, \beta}$-norm on $C^{2, \beta}(M)$. Besides $(L-\lambda I)^{-1}$ is strongly positive, i.e. $w \in C^{\beta}(M), w \geq 0, w \not \equiv 0$, implies $(L-\lambda I)^{-1} w>0$ on $M$. So it extends uniquely to a compact map, still denoted 
$(L-\lambda I)^{-1}$ from $C(M)$ into $C(M)$, which is still strongly positive (cf. [Am]). For each $w \in C(M),(L-\lambda I)^{-1} w$ is a weak solution of $L u-\lambda u=w$.

The nonlinear compact and order preserving operator $v \rightarrow(L-\lambda I)^{-1}\left(|H||v|^{\alpha}\right)$ : $C(M)^{+} \rightarrow C(M)^{+}$leaves invariant the order interval $\left[\underline{t} u_{1}, \bar{t} u_{1}\right] \subset C(M)$, so it has a fixed point $u \in C(M)$, with $0<\underline{t} u_{1} \leq u \leq \bar{t} u_{1}$, i.e. $u=(L-\lambda I)^{-1}\left(|H||u|^{\alpha}\right)$, hence by a classical bootstrap argument $u \in C^{\infty}(M)$; in particular $u$ is a classical positive solution of (3.2) (cf. [Am] in the Neumann case for details). Finally the nonlinearity $|H| t^{\alpha}$ in (3.2) is such that $t^{\alpha} / t$ is strictly decreasing in $t>0$; hence $u$ is unique [L, Bere]. As $f=u^{2 /(n+1)}$ we obtain the uniqueness.

The situation seems more complicated when $H>0$.

THEOREM 3.3. Let $M=\left(M_{m}, g\right)$ be compact and connected. Suppose $N=$ $\left(N_{n}, h\right)$ of constant positive curvature $H$ and assume $n \geq 3$. Let $\lambda_{1}$ denote the principal eigenvalue of $-4 n \Delta_{g} /(n+1)+R$. Then for each $\lambda$ in some interval $\left(\lambda_{1}, \lambda_{1}+\delta\right)$ there exists a weight $f=f(\lambda)$ such that $M \times_{f} N$ has $\lambda$ as scalar curvature. No constant $\leq \lambda_{1}$ may be curvature of $M \times_{f} N$ for any $f$.

PROOF. If $u>0$ on $M$ is a solution of (3.2) with $H>0$, then (3.3) gives that $\lambda>\lambda_{1}$ is a necessary condition.

Denote $\|u\|=\max _{M}|u|, v=u /\|u\|^{2}$ for $u \neq 0$, i.e. $u=v /\|v\|^{2}$. We will obtain solutions as a bifurcation from infinity near $\lambda=\lambda_{1}$ (cf. [R-2]). Multiplying our equation (3.2) by $1 /\|u\|^{2}$, replacing by $v$, we are reduced to finding positive solutions of

$$
L v+a v=\lambda v-H\|v\|^{2(1-\alpha)} v^{\alpha}+a v
$$

where $\lambda \in \mathbf{R}$ is a parameter and $a \in \mathbf{R}^{+}$is a fixed number chosen big enough so that the operator $(L+a I): C^{2, \beta}(M) \rightarrow C^{\beta}(M)$ is an isomorphism with positive inverse. Hence, as in the preceding proof, $(L+a I)^{-1}: C(M) \rightarrow C(M)$ is a linear compact strongly prositive operator such that $(L+a I)^{-1} v>0$ on $M$ if $v \geq 0$, $v \not \equiv 0$ on $M$.

We now search for pairs $(\lambda, v) \in \mathbf{R} \times C(M)$ with $v>0$ on $M$, solutions of

$$
v=\mu(L+a I)^{-1} v-H(L+a I)^{-1} f(v)
$$

where $\mu=\lambda+a, H>0$, and $f(v)=\|v\|^{2(1-\alpha)}|v|^{\alpha}$. We easily see that $H(L+a I)^{-1} f(v)=o(\|v\|)$ in $C(M)$ for $\|v\|$ near 0 . Moreover from $L u_{1}=\lambda_{1} u_{1}$, it follows that $\mu_{1}=\lambda_{1}+a$ is a simple characteristic value of the compact map $(L+a I)^{-1}$. Then by the Rabinowitz bifurcation theorem [R-1], there exists a maximal connected closed subset $C_{\mu_{1}}$ of

$$
S=\operatorname{adh}_{\mathbf{R} \times C(M)}\{(\lambda, v) \text { solutions of (3.5) with } v \neq 0\}
$$

such that $\left(\mu_{1}, 0\right) \in C_{\mu_{1}}$ and either

(i) $C_{\mu_{1}}$ is bounded in $\mathbf{R} \times C(M)$ or

(ii) $C_{\mu_{1}}$ meets $(\hat{\mu}, 0)$ with $\mu_{1} \neq \hat{\mu} \in\{\lambda+a ; \lambda$ eigenvalue of $L\}$.

We write $v=\gamma u_{1}+w$, with $\gamma=v\left(x_{o}\right)$, where $u_{1}\left(x_{o}\right)=\left\|u_{1}\right\|$ and $w\left(x_{o}\right)=0$. For $\xi>0,0<\eta<1$, the open sets in $\mathbf{R} \times C(M): K_{\xi, \eta}^{+}, K_{\xi, \eta}^{-}$defined by

$$
K_{\xi, \eta}^{ \pm}=\left\{(\mu, v) \in \mathbf{R} \times C(M) ;\left|\mu-\mu_{1}\right|<\xi, \pm \gamma<\eta\|v\|\right\}
$$

satisfy both $K_{\xi, \eta}^{ \pm} \cap C_{\mu_{1}} \neq \varnothing$ for $\xi$ small enough as in Theorem 1.25 in [R-1]. Consequently the maximal connected closed subset (or continuum) of $C_{\mu_{1}}$ contained 
in $\left\{\left(\mu, \gamma u_{1}+w\right) ; \gamma>0\right\} \cup\left\{\left(\mu_{1}, 0\right)\right\}$ is nontrivial; we denote it as $C_{\mu_{1}}^{+}$. Besides from $H(L+a I)^{-1} f(v)=o(\|v\|)$ we deduce that $\|w\|=o(\gamma)$ near $\gamma=0$, so $v=\gamma u_{1}+w \in C_{\mu_{1}}^{+}$is (strictly) positive on $M$ for $\gamma>0$ small enough.

We have then obtained a "branch" of $C(M)$-solutions $(\mu, v), v>0$ on $M$ of (3.5). Going back to $u=v /\|v\|^{2}, \lambda=\mu-a$, we have weak $C(M)$-solutions of (3.2): $(\lambda, u)$ for $\lambda$ near $\lambda_{1}, u>0$ on $M,\{\|u\|\}$ unbounded. By a regularity argument, each $u$ is a $C^{\infty}$ classical solution and by the necessary condition we have $\lambda>\lambda_{1}$.

REMARK 1. The nonlinearity $v \rightarrow(L+a I)^{-1} f(v)=\|v\|^{2(1-\alpha)}(L+a I)^{-1}\left(|v|^{\alpha}\right)$ is not always differentiable for $\|v\|$ near 0 , so we cannot directly apply the results in $[\mathbf{R}-\mathbf{1}, \mathbf{2}]$.

REMARK 2. The operator $F(\mu, v)=(L+a I)^{-1}(\mu v-H f(v)), H>0$, generally transforms a positive $v$ into a nonpositive $F(\mu, v)$, so some known results on maps of cones into cones [L, T] do not apply. More precisely, when $N$ is 3-dimensional, (3.2) can be written as

$$
\left(-3 \Delta_{g}+R I-\lambda I\right) u=-H
$$

and Theorem 3.3 is a consequence of the antimaximum principle (cf. [C-P]) which says that $\lambda>\lambda_{1}$ and near $\lambda_{1}$ implies that negative data $-H$ on $M$ gives a positive solution $u$, on the contrary of the maximum principle.

Let us denote

$$
C_{\infty}=\left\{(\lambda, u) ; u \geq 0, u \neq 0,\left(\lambda+a, u /\|u\|^{2}\right) \in C_{\mu_{1}}^{+}\right\} \subset \mathbf{R} \times C(M)^{+},
$$

the nonnegative weak $C(M)$-solutions bifurcating from infinity. We know now that $H>0$ fixed implies that $u>0$ for $\lambda>\lambda_{1}, \lambda$ near $\lambda_{1}$. We can say more on $\|u\|$ in $c_{\infty}$

THEOREM 3.4. Let $M, N$ and $\lambda_{1}$ be as in Theorem 3.3. For any $0<\varepsilon<A$, the set $\left\{(\lambda, u) \in C_{\infty} ; \lambda_{1}+\varepsilon \leq \lambda \leq \lambda_{1}+A\right\}$ is bounded in $\mathbf{R} \times C(M)$.

PROOF. Suppose not. Then there exists a sequence $\left(\lambda_{n}, u_{n}\right) \in \mathcal{C}_{\infty}$ with $\lambda_{n} \in$ $\left[\lambda_{1}+\varepsilon, \lambda_{1}+A\right], u_{n} \geq 0$ on $M, u_{n} \neq 0, \lim _{n} \lambda_{n}=\lambda$ and $\lim _{n}\left\|u_{n}\right\|=+\infty$, which satisfies

$$
u_{n} /\left\|u_{n}\right\|=\left(\lambda_{n}+a\right)(L+a I)^{-1}\left(u_{n} /\left\|u_{n}\right\|\right)-H(L+a I)^{-1}\left(u_{n}^{\alpha} /\left\|u_{n}\right\|\right) .
$$

$(L+a I)^{-1}: C(M) \rightarrow C(M)$ being compact we may suppose, up to a subsequence, that $\lim _{n}(L+a I)^{-1}\left(u_{n} /\left\|u_{n}\right\|\right)=\tilde{u}$ in $C(M)$. From $\lim _{n}\left(u_{n}^{\alpha} /\left\|u_{n}\right\|\right)=0$ in $C(M)$, we obtain that $u_{n} /\left\|u_{n}\right\|$ tends to some $u$ in $C(M), u \geq 0,\|u\|=1$, so we have

$$
u=(\lambda+a)(L+a I)^{-1} u
$$

i.e., by regularity properties, $-4 n \Delta u /(n+1)+R u=\lambda u, v \geq 0, u \neq 0$, hence $u=u_{1}$ and $\lambda=\lambda_{1}$ by uniqueness. This contradicts $\lambda \geq \lambda_{1}+\varepsilon$.

REMARK. If the set $C_{\infty}$ meets $(\hat{\mu}, 0) \in \mathbf{R} \times C(M)$ so does $C_{\mu_{1}}^{+}$. Then $\hat{\mu} \neq \mu_{1}$ and $\hat{\mu}$ is necessarily a characteristic value of $(L+a I)^{-1}$ (cf. $\left.[\mathbf{R}-\mathbf{1}]\right)$. Hence $\hat{\mu}=\hat{\lambda}+a$, $\hat{\lambda}>\lambda_{1}, \hat{\lambda}$ an eigenvalue of $-4 n \Delta /(n+1)+R$. We would then have a sequence $\left(\mu_{k}, v_{k}\right) \in C_{\mu_{1}}^{+}$, such that $\lim _{k} \mu_{k}=\hat{\mu}, \lim _{k} v_{k}=\lim _{k} u_{k} /\left\|u_{k}\right\|^{2}=0$ in $C(M)$, $v_{k} \neq 0$, i.e. $\left(\lambda_{k}, u_{k}\right) \in C_{\infty}$, with $\lambda_{k}=\mu_{k}-a \geq \lambda_{1}+\varepsilon,\left\{\lambda_{k}\right\}$ bounded and $\lim _{k}\left\|u_{k}\right\|=+\infty$, contradicting Theorem 3.4. Then $C_{\infty}$ never meets some $(\lambda, 0)$.

For $n=3, H>0$ we are able to characterize the case that for any $\lambda>\lambda_{1}$ there is a $u(\lambda)>0$ on $M$ with $(\lambda, u(\lambda)) \in C_{\infty}$. 
THEOREM 3.5. Let $M, N$ and $\lambda_{1}$ be as in Theorem 3.3 with $\operatorname{dim} N=3$. Then any constant $\lambda \in\left(\lambda_{1}, \infty\right)$ is scalar curvature of $M \times_{f} N$ for some weight $f$ if and only if the scalar curvature $R$ on $M$ is constant.

PROOF. If $R$ is constant and $\lambda>\lambda_{1}, u(\lambda)=H /\left(\lambda-\lambda_{1}\right)$ is a solution of (3.7), hence $f=u^{1 / 2}$ is a constant weight which gives $\lambda$ as scalar curvature on $M \times_{f} N$.

Conversely, suppose that for each $\lambda>\lambda_{1}$ there corresponds a weight $f$ i.e. a solution $u$ of $(3.7)$. This means for $\lambda=\lambda_{k}(k>1)$ an eigenvalue of the selfadjoint operator $-3 \Delta_{g}+R$, that the second member in equality (3.7) is orthogonal to the corresponding eigenspace. Hence $H$ is orthogonal to all eigenfunctions except the principal one $u_{1}$, so $H=t u_{1}$ with $t>0$ by the completeness of an orthonormal system of eigenfunctions, i.e. $u_{1}$ is constant and (3.7) with $u=u_{1}$ gives $R$ constant.

Ejiri proves in [E-2] that there exist countable immersions of $S^{1} \times S^{n}$ into $S^{n+2}$ such that $S^{1} \times S^{n}$ is a warped product of constant scalar curvature $n(n+1)$ with respect to the induced metric.

On one hand, if we consider equation (2.1) in $S^{1} \times S^{n}$, we have $R=0$ on $S^{1}$ and $H=n(n-1)$ on $S^{n}$ with the usual metric. So [E-2] gives a countable number of positive solutions of

$$
-\frac{4 n}{n+1} u^{\prime \prime}+H u^{(n-3) /(n+1)}=n(n+1) u .
$$

In particular for $n=3$, this equation becomes

$$
-3 u^{\prime \prime}-12 u=-H
$$

with $H=6$.

On the other hand, equation (3.7) reduces on $S^{1} \times S^{3}$ to

$$
-3 u^{\prime \prime}-\lambda u=-H \text {. }
$$

This last equation has a unique solution $u=u(\lambda)$ for $\lambda>\lambda_{1}, \lambda$ not an eigenvalue. But $\lambda=12$ is an eigenvalue of $(-3)$ times the laplacian on $S^{1}$, the eigenvalues being $\left\{\lambda_{k}=3(k-1)^{2}, k=1,2, \ldots\right\}$, so the countable solutions of (3.9) given in [E-2] appear for $\lambda=\lambda_{3}$. But then we have uncountable positive solutions of (3.10) for $\lambda=\lambda_{k}, k>1$, they are

$$
v=H / \lambda_{k}+t u_{k}
$$

where $-3 u_{k}^{\prime \prime}=\lambda_{k} u_{k}$ and $|t|$ small enough so that $v$ is positive. We have proved

THEOREM 3.6. Let $M, N$ and $\lambda_{1}$ be as in Theorem 3.3 with $\operatorname{dim} M=1$ and $\operatorname{dim} N=3$. Given an eigenvalue $\lambda_{k}, k>1$, of $-3 \Delta_{g}$ there exist uncountable weights $f$ such that $M \times_{f} N$ has $\lambda_{k}$ as scalar curvature.

REMARK. The $u=f^{2}$ from this theorem are secondary bifurcations of the branch $\{H / \lambda\}$ of solutions considered up to now, at points $\lambda=\lambda_{k}$. In the case $M=S^{1}$ and $N=S^{n}$ with $n>3$, the curvature on $S^{1} \times_{f} S^{n}$ given in [E-2] is $n(n+1)$ with $u=f^{(n+1) / 2}$ a solution of (3.8). This constant $n(n+1)$ is far from the scalar curvatures obtained from Theorem 3.3 which are near zero, i.e. our solutions of (2.1) and those in [E-2] on $S^{1} \times S^{n}$ seem to be of different type.

Theorem 3.2 still holds for $N$ of dimension 2 with a different proof inspired from [C-R-T]. 


\section{REFERENCES}

[Am] H. Amann, Fixed point equations and nonlinear eigenvalue problems in ordered Banach spaces, SIAM Rev. 18 (1976), 620-709.

[Au1] T. Aubin, Nonlinear analysis on manifolds. Monge-Ampère equations, A Series of Comprehensive Studies in Mathematics 252, Springer, Berlin.

[Au2] _- Métriques Riemanniennes et courbure, J. Differential Geom. 4 (1970), 383-424.

[Bere] H. Berestycki, Le nombre de solutions de certains problèmes semi-linéaires elliptiques, J. Funct. Anal. 40 (1981), 1-29.

[B-G-M] M. Berger, P. Gauduchon, and E. Mazet, Le spectre d'une variété Riemannienne, Lecture Notes in Math., vol. 194, Springer, 1971.

[B-O] R. L. Bishop and B. O'Neill, Manifolds of negative curvature, Trans. Amer. Math. Soc. 145 (1969), 1-49.

[C-P] Ph. Clément and L. A. Peletier, An anti-maximum principle for second-order elliptic operators, J. Differential Equations 34 (1979), 218-229.

[C-R-T] M. G. Crandall, P. H. Rabinowitz, and L. Tartar, On a Dirichlet problem with a singular nonlinearity, MRC Report 1680, 1976.

[E-1] N. Ejiri, A negative answer to a conjecture of conformal transformations of Riemann manifolds, J. Math. Soc. Japan 33 (1981), 261-266.

[E-2] _ Some compact hypersurfaces of constant scalar curvature in a sphere, J. Geom. 19 (1982), 197-199.

[L] P. L. Lions, On the existence of positive solutions of semilinear elliptic equations, SIAM Rev. 24 (1982), 441-467.

[M] J. Moser, On a nonlinear problem in differential geometry, Dynamical Systems, M. M. Peixoto (ed), Academic Press, New York, 1973, pp. 273-280.

[R1] P. H. Rabinowitz, Some global results for nonlinear eigenvalue problems, J. Funct. Anal. 7 (1971), 487-513.

[R2] _ On bifurcation from infinity, J. Differential Equations 14 (1973), 462-475.

[T] E. E. L. Turner, Positive solutions of nonlinear eigenvalue problems, C.I.M.E. Varenna, 1974, pp. 212-239.

[Y] H. Yamabe, On a deformation of Riemannian structures on compact manifolds, Osaka Math. J. 12 (1960), 21-37.

[Z] S. Zucker, $L_{2}$ cohomology of warped products and arithmetic groups, Invent. Math. 70 (1982), 169-218.

Departamento de matemática, FCeyn, Universidad de Buenos Aires, Buenos Aires, ARGENTINA

instituto Argentino de Matemática (CONiCet), Viamonte 1636, 1055 Buenos Aires, Argentina 